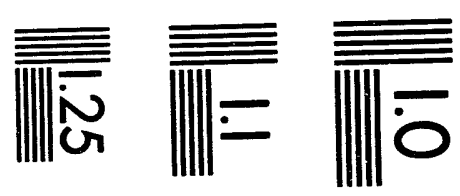

$$
\begin{aligned}
& \text { IIIID }
\end{aligned}
$$

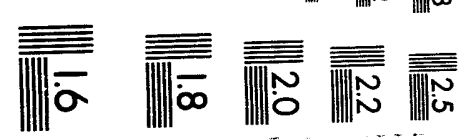



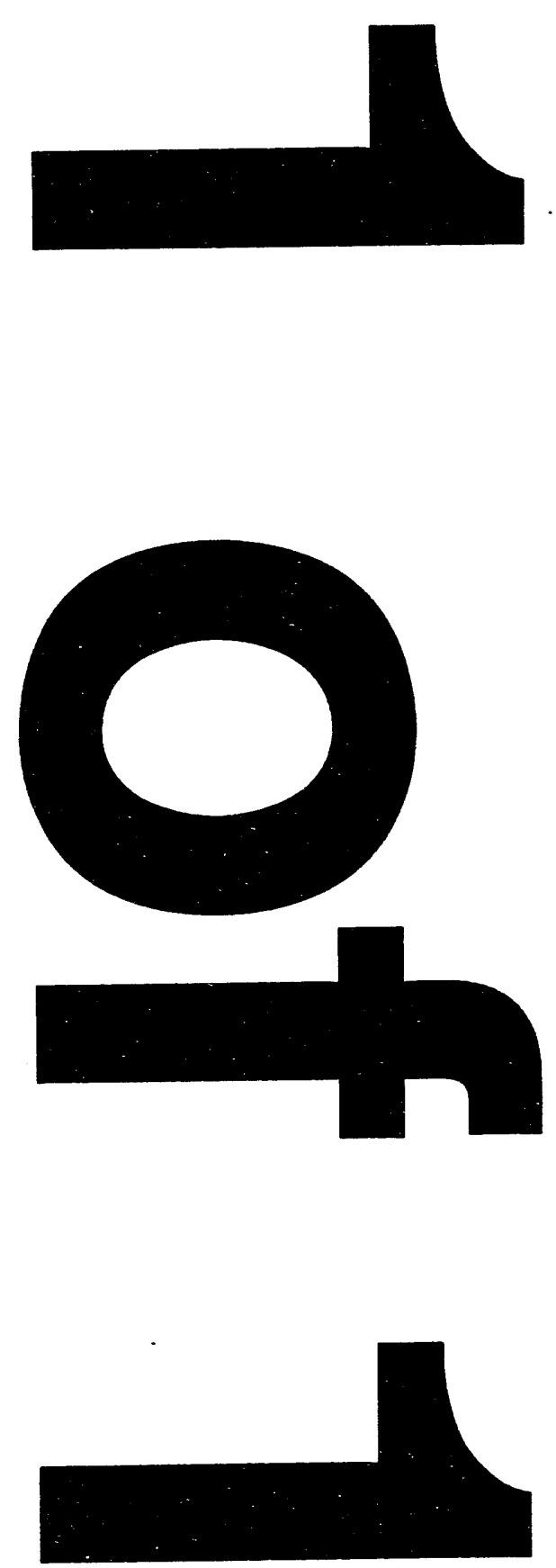
DOE Report No. DOE/PC $\not 0284-12$

\section{OEC 23}

November 1993

Quarterly Report

for

Grant No. DE-FG22-90PC90284

entitled

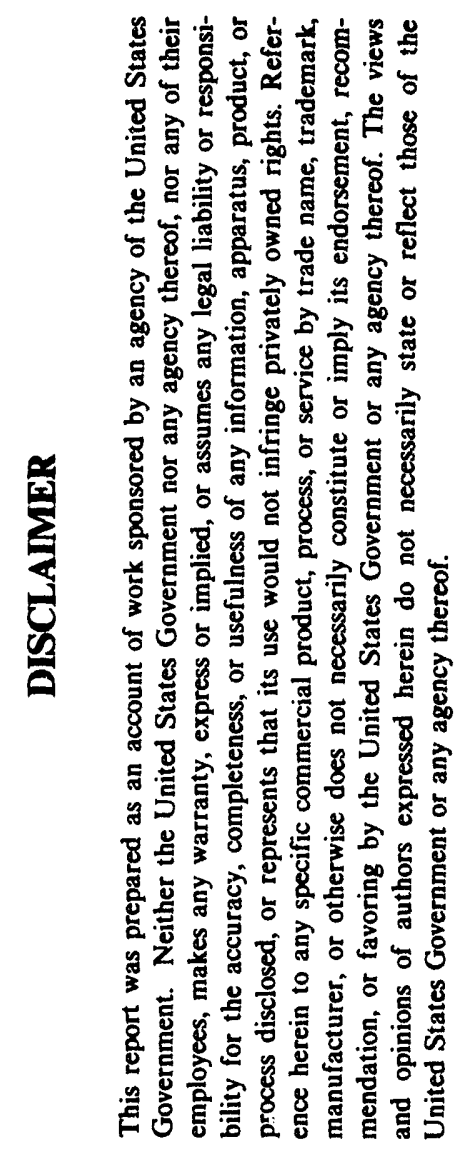

\section{Molecular Accessibility in Solvent Swelled Coals}

Lowell D. Kispert, Principle Investigator

Department of Chemistry

The University of Alabama

Tuscaloosa, AL 35487-0336

US/DOE Patent clearance is not required prior to the publication of this document. 


\section{PREVIOUS WORK COMPLETED}

An EPR technique developed in this lab ${ }^{1-3}$ has been used to determine the pore size and number distribution changes after swelling the coal samples with various solvents. Stable nitroxide radical spin probes of different sizes, shapes and reactivity are dissolved in an appropriate solvent, the coal sample is then added to the resulting solution, stirred overnight at elevated temperature, filtered, washed with a non swelling solvent to eiiminate any spin probes that are not trapped in the pores and the spin concentration is measured. Comparing these spin probe measurements to DRIFT data have shown ${ }^{4}$ that the relative number distribution of acidic functionalities can be accurately predicted by the spin probe method. The spin probe method had also been used to predict $^{+5}$ the increase in elongated voids in Pittsburgh No. 8 (APCS No. 4) upon swelling with pyridine in agreement with independent SANS data. NMR relaxation data show ${ }^{3}$ that it is possible to deduce the pore (accessibility) distribution as a function of size (up to $6 \mathrm{~nm}$ ). It has also been possible by variable temperature ${ }^{1-3}$ and ENDOR measurements ${ }^{6}$ to determine the presence of hydrogen bonding as a function of pore shape and size. The advantage of the EPR method is that it permits molecules of selected shape, size and reactivity to be used as probes of accessible regions of coal, thus providing insormation of importance to the diffusion and reactivity behavior of catalysts in coal.

To expand the information base on molecular accessibility in solvent swelled coal, Argonne Premium Coal Samples (APCS) were swelled in polar, basic solvents before and after moisture loss and upon air oxidation. So far studies have been reported ${ }^{7.8}$ on the changes in pore size distribution as a function of temperature when polar basic swelling solvents are used. Additional 
studies employing EPR spin probe techniques performed on the breaking up of the hydrogen bonding between bedding planes $^{9.10}$ were later confirmed by magnetic resonance imaging at Argonne National Lab and the University of Illinois.

A paper $^{11}$ has recently appeared in the journal, Fuel on the micropore wall chemistry during swelling. Studies of spin probe retention with increasing spin probe polarity, provided valuable insight into the hydrogen bond cross-link density and oxygen functionality of APCS coals.

Eight Argonne Premium Coal Samples (APCS) were weathered in air for 8 days and the structural and chemical changes that occurred upon swelling with toluene and pyridine were examined by our EPR spin probe method. The large structural changes were attributed to collapse of the coal structure. The results were reported ${ }^{12}$ at the ACS Fuel Chemistry Division Annual meeting in Washington, DC, August 23-26, 1992 in the Symposium on Analytical Techniques for Characterizing Coal and Coal Conversion Products.

The EPR spin probe technique was used to examine the swelling behavior of $2 \%$ to $12 \%$ cross-linked polystyrene-divinylbenzene (PSDVB) copolymers to gain insight into the molecular accessibility of covalently cross-linked coal. Since these copolymers can be highly cross-linked with covalent interactions, without the presence of hydrogen bonding or other polar interactions prominent in coal, they are valuable models for studying the molecular accessibility as a function of only the covalently cross-linked character in coal. 
Because the polymers contained no polar functional groups, selective retention of I, TEMPO $(\mathrm{R}=$ $\mathrm{OH}$, a spherical molecule with a hydrogen bond site) over VIII, TEMPO ( $\mathrm{R}=\mathrm{H}$, a spherical molecule with no hydrogen bonding site) was not observed. Pyridine was determined to be a much better swelling solvent than toluene for PSDVB, however, toluene was shown to be a much more effective solvent for the inclusion of spin prob $\epsilon_{s}$ in the macromolecular structure of PSDVB copolymers following a cyclohexane wash. It was demonstrated that most of the spin probe retention was due to the intercalation of the guest molecules into the structure of the copolymers and not to any inclusion of spin probes in pre-existing pores accessible from the surface of the polymer beads. The extent of hydrogen bonding in coal was inferred to have a much greater impact on its swelling properties than its covalently cross-linked character. However, significant amounts of spin probe retention can be achieved in covalent cross-linked materials. ${ }^{13}$

The effect of weathering (oxidation and dehydration upon exposure to air) on the molecular accessibility of potential catalysts was studied by the EPR spin probe technique. Fresh samples of all 8 APCS coals were exposed to air for periods up to 36 days. Weathering produced significant effects on the retention of spin probes in most of the APCS coals under $91 \%$ carbon (dmmf). It was determined that the lower ranked coal (Beulah Zap and Wyodak) underwent a structural collapse which precluded retention of even spin probe VIII. However, medium ranked coals exhibited improved retention upon weathering when swelled in toluene. Swelling with pyridine opened up small pores for $81 \%-86 \%$ carbon which is not observed for swelling with toluene. Changes in coal structure were successfully followed by the EPR spin probe method. ${ }^{14}$ 
A detailed analysis of the data collected from the swelling of coals oxidized in a moisture free environment was completed to differentiate between weathering and oxidation. Eight vacuum dried APCS coals were oxidized in an enclosed, pure oxygen, moisture free environment, and the effects of oxidation alone on coal structure were studied by the intercalation of EPR spin probes. The data shows a factor of 5 increase in spin probe retention for some coals oxidized in $\mathrm{O}_{2}$ versus air, suggesting a large increase in oxidized material. Particular care was taken during the swelling procedures to avoid exposure of the coal samples to air or moisture. EPR spectra were then obtained for these 300 samples. ${ }^{15}$

The effects of short term exposure of Illinois \#6 swelled in toluene to both argon and oxygen on the retention of spin probe VI were examined last quarter. It is clear from these studies that significant structural changes in Illinois \#6 can occur in as little as 30 seconds of exposure to a dry gas environment. This suggests that the design of an experiment to study coal properties must consider the effect of a drying and oxidizing atmosphere. ${ }^{16}$

\section{SUMMARY OF CURRENT ACTIVITIES}

This quarter, experiments were performed on the use of binary swelling solvents in molecular accessibility in coal conversion. Preliminary results were reported in an invited paper in the Symposium on Coal Conversion at the 45th ACS Southeast Regional meeting held in Johnson City, Tennessee by David Tucker. These experiments consisted of accessibility measurements of spin probe VII (TEMPAMINE) in Toluene swelled Illinois \#6 APCS coal. The toluene was spiked with amounts of pyridine which ranged in concentration from $500 \mathrm{ppm}$ to $10 \%$. The 
experiments were done in triplicate to gain information about the experimental error involved in the procedure. It was shown that oscillations occur in the concentration of spin probe retained as the amount of pyridine that is added to the swelling solvent is increased. These oscillations decrease in intensity as the concentration of pyridine in the solvent solution is increased up to $2 \%$ pyridine $(0.2 \mathrm{mLs}$ pyridine in $10 \mathrm{mLs}$ toluene). From a $2 \%$ pyridine concentration to a $5 \%$ concentration, there is no significant change in the retention of spin probe VII. An increase in retention is observed when the concentration of pyridine is increased to $6 \%$ and $7 \%$ successively, followed by a large decrease at $8 \%$ and $9 \%$ pyridine. The largest changes in spin probe retention are observed for concentrations of pyridine less than $0.5 \%$. A three fold increase in spin probe retention is observed upon the addition of $500 \mathrm{ppm}$ pyridine in the toluene swelling solvent, which solvert

indicates that small amounts of a strong swelling could be used to improve molecular accessibility in coals swelled in an otherwise weak swelling solvent.

\section{STUDIES PLANNED FOR NEXT QUARTER}

Next quarter, further examination of the use of binary swelling solvent will be completed. Spin probe VIII will be used to obtain information about structural changes in Illinois \#6 and these experiments will be performed on lower ranked coals so that a more complete picture of the binary swelling system might be obtained.

\section{REFERENCES}

1. Wu S. K; Kispert, L. D. Fuel 1981, 64, 1681-1686 
2. Kispert, L. D. ; Cooray, L. S.; Wu, S. K. Prepr. Pap. Am. Chem. Soc., Div. Fuel. Chem. 1988, 32, 286-292.

3. Cooray, L. S.; Kispert, L. D.; Wu, S. K. Prepr. Pap. Am. Chem. Soc., Div. Fuel. Chem. 1988, $33,32-37$.

4. Goslar, J.; Kispert, L. D. Energy and Fuels 1989, 3, 589-594.

5. Goslar, J.; Cooray, L. S.; Kispert, L. D. Fuel 1989, 68, 1402-1407.

6. Goslar, J., Kispert, L. D. Fuel 1990, 69, 564-569.

7. Spears, R.; Goslar, J.; Kispert L. D. in "Magnetic Resonance of Carbonaceous Solids," Botto, R., and Sanada, Y. Eds., ACS Symposiurn Series No. 229, 1993.

8. Spears, R.; Kispert, L. D.; Piekara-Sady, L. Prepr. Pap. Am. Chem. Soc., Div. Fuel. Chem. 1991, 36, 29.

9. Spears, R.; Sady, W.; Kispert, L. D. Prepr. Pap. Am. Chem. Soc., Div. Fuel. Chem. 1991, 36, 1277

10. Spears, R.; Kispert, L. D.; Piekara-Sady, L. Fuel 1992, 7l, 1003.

11. Spears, D. R.; Sady, W.; Kispert, L. D. Fuel 1993, 72, 1225

12. Sady, W.; Kispert, L. D.; Spears, D. R. Prepr. Pap. Am. Chem. Soc., Div. Fuel. Chem. 1992, 37,1151

13. Spears, D. R.; Sady, W.; Tucker, D; Kispert, L. D. Energy and Fuels, In Press.

14. Sady, W.; Tucker, D.; Kispert, L.. D.; Spears, D. R. Prepr. Pap. Am. Chem. Soc., Div. Fuel. Chem. 1993, 38, 1323

15. Tucker, D.; Kispert, L. D. Prepr. Pap. Am. Chem. Soc., Div. Fuel. Chem. 1993, 38, 1330.

16. Tucker, D.; Kispert, L. D. Prepr. Pap. Am. Chem. Soc., Div. Fuel. Chem. 1993, 38, 1335. 

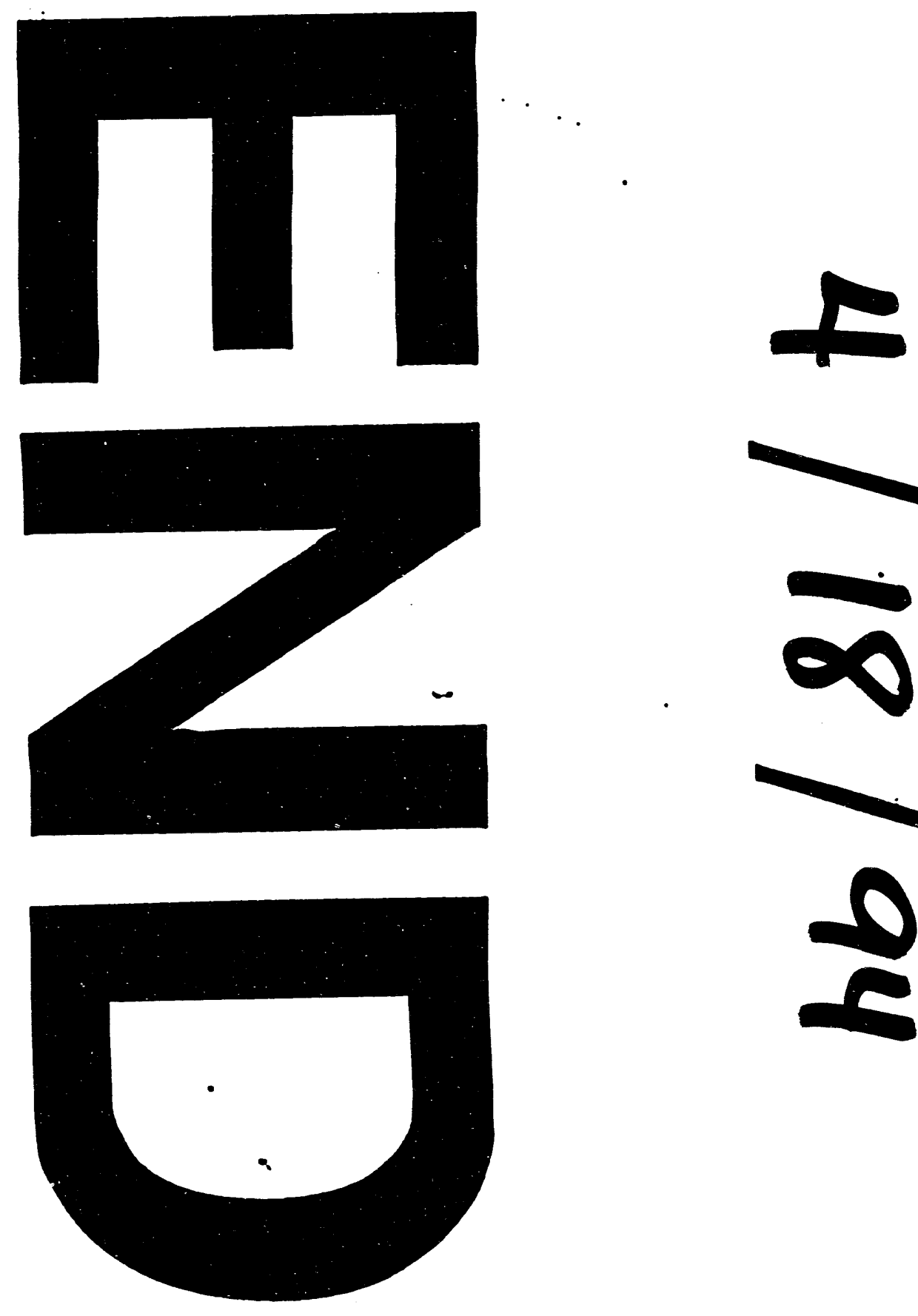
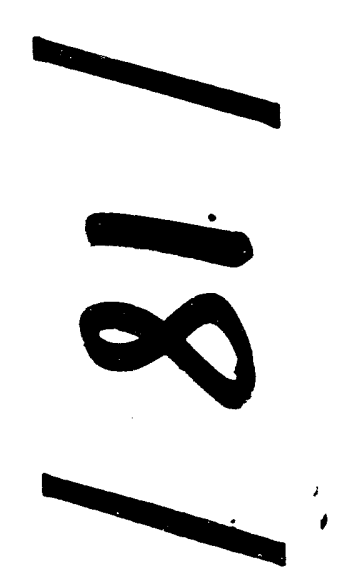

$\geq$

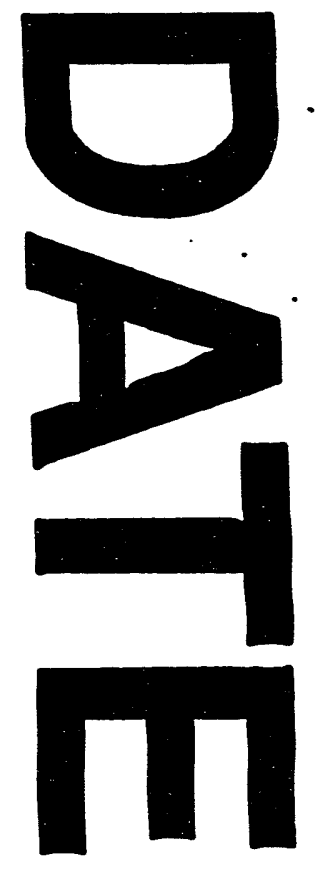


